\title{
Separation-free $\mathrm{TiO}_{2}$-graphene hydrogel with 3D network structure for efficient photoelectrocatalytic mineralization
}

\author{
Xianjie Chen ${ }^{\mathrm{a}}$, Qian Chen ${ }^{\mathrm{b}}$, Wenjun Jiang ${ }^{\mathrm{a}}$, Zhen Wei ${ }^{\mathrm{a}}$, Yongfa Zhu ${ }^{\mathrm{a} *}$
}

\author{
a Department of Chemistry, Tsinghua University, Beijing, 100084, P. R. China \\ ${ }^{\mathrm{b}}$ College of Chemistry and Chemical Engineering, Xiamen University, Xiamen, 361005, P. R. China
}

\section{Corresponding Author}

*E-mail: zhuyf@ tsinghua.edu.

\begin{abstract}
Herein, $\mathrm{TiO}_{2}$-graphene hydrogel (TGH) electrodes with 3D network structure were designed and fabricated successfully via the one-pot method and the photoelectrocatalytic mineralization ability over TGH electrodes was studied in a dynamic system. TGH electrodes showed interconnected open-framework and large specific surface area, which greatly improved the adsorption capacity for organic pollutants. Most interestingly, TGH electrodes exhibited remarkably superior photoelectrocatalytic degradation activity for MB and phenol, the removal rate was 3 times higher than that of P25. In this process, strong oxidizing $\mathrm{h}^{+}$ and $\cdot \mathrm{OH}$ radicals worked as the main active groups, contributed to the deep mineralization ability for organic pollutants over TGH electrodes. The efficient photoelectrocatalytic mineralization ability of TGH electrodes should mainly benefit from the synergism between adsorption-enrichment and in-situ photoelectrocatalytic oxidation, and the extremely fast transfer for photogenerated electrons on graphene hydrogel. Besides, TGH
\end{abstract}


electrodes not only took advantage of the efficient activity of nano-photocatalysts, but also possessed the separation-free feature. Overall, a facile route to construct 3D network-structure hydrogels with efficient photoelectrocatalytic mineralization ability was revealed and can potentially be utilized in water purification.

Keywords: separation-free, 3D network-structure, photoelectrocatalytic, deep mineralization

\section{Introduction}

Over the past decades, semiconductor photocatalytic oxidation technology has been intensively investigated in wastewater treatment [1-6], which should be mainly ascribed to its high effectiveness and almost no harmful secondary products [7-11]. Among varied photocatalysts, $\mathrm{TiO}_{2}$ has been proved to be one of the most promising photocatalysts because of whose deep mineralization ability, excellent chemical stability, and photocorrosion resistance, and notable advances have been made in $\mathrm{TiO}_{2}$ photocatalytic oxidation technology[12-17]. However, the recovery of photocatalyst materials has been the biggest problem restricting their practical application as most photocatalysts including $\mathrm{TiO}_{2}$ are nanoscale[18]. Therefore, it is urgently needed to develop a new type of photocatalyst with the separation-free feature and high activity.

Fabrication of hydrogel-based photocatalysts with 3D network structure is regarded as an extremely effective method, because it not only has the advantage of the efficient activity in nanomaterials, but also possesses the separation-free feature [19, 20]. 3D network-structure hydrogel-based photocatalysts show large specific surface area, which enables them to exhibit strong adsorption-enrichment for pollutants and more active sites to be exposed for accelerating the surface catalytic reaction[21-24]. In addition, the 3D network structure creates convenient channels for photogenerated carrier transfer, and the utilization of incident photons could be maximized via the multireflection within the interconnected open-framework [25, 26]. In recent years, 3D network-structure PANI/TiO $2, \mathrm{PANI} / \mathrm{g}-\mathrm{C}_{3} \mathrm{~N}_{4}, \mathrm{~g}-\mathrm{C}_{3} \mathrm{~N}_{4} / \mathrm{Agar}$, and $\mathrm{g}-\mathrm{C}_{3} \mathrm{~N}_{4} / \mathrm{SiO}_{2}$ hydrogel have been synthesized via a facile route by our group $[19,20,27,28]$. The nanoparticles were 
located onto the 3D network-structure matrix of the hydrogels, which means that the 3D network-structure photocatalysts has the separation-free feature. More importantly, benefiting from the synergism between adsorption-enrichment and in-situ photocatalytic oxidation, the 3D network-structure photocatalysts exhibited enhanced photocatalytic degradation activity and deep mineralization ability. During this process, pollutants were first adsorbed and enriched by the 3D network-structure hydrogel and then in-situ oxided.

The substrate materials of hydrogel play a key role in constructing efficient 3D network-structure hydrogel-based photocatalysts. In this regard, graphene hydrogels have been proved to be a preferred choice, which should be attributed to its 3D network structure, large specific surface area and high adsorptionenrichment capacity for pollutants [29-33]. Graphene hydrogels could be synthesized conveniently via the self-assembly of graphene oxide nanosheets with the $\pi-\pi$ stacking between nanosheets[34, 35]. Up to now, graphene hydrogels have been widely explored and applied in the field of photocatalytic degradation. Thanks to the strong adsorption ability and extremely fast transfer for photogenerated carriers over graphene, the graphene-based hydrogel photocatalysts showed efficient activity [25, 26, 29, 36-41]. Zhang's group synthesized mesoporous $\mathrm{TiO}_{2}$ with (001) facets exposed on graphene aerogel $\left(\mathrm{TiO}_{2} / \mathrm{GAs}\right)$ via a one-step hydrothermal method[42]. $\mathrm{TiO}_{2} / \mathrm{GAs}$ possessed hierarchically porous structure and a high surface area, besides, exhibited an efficiently recyclable photocatalytic degradation activity for organic pollutants. What's more, Jiang's group also reported a facile hydrothermal route toward 3D porous $g-\mathrm{C}_{3} \mathrm{~N}_{4} /$ graphene oxide aerogel (CNGA) via the coassembly of $\mathrm{g}_{-} \mathrm{C}_{3} \mathrm{~N}_{4}$ nanosheets and graphene oxide nanosheets[43]. CNGA showed high photocatalytic activity for degrading methyl orange. However, it remains a challenge to prepare graphene-based hydrogel photocatalysts at relatively low temperature and meet the deep mineralization ability for organic pollutants over them, which greatly restricted their practical application.

Herein, $\mathrm{TiO}_{2}$-graphene hydrogel (TGH) electrodes with 3D network structure were designed and 
fabricated successfully via a facile one-pot method at $90^{\circ} \mathrm{C}$ and the photoelectrocatalytic degradation of methylene blue (MB) and phenol over TGH electrodes was studied in a dynamic system. The effects of morphologies, photoelectric performance, and active groups of TGH on photoelectrocatalytic activity were elucidated in detail. TGH electrodes possess large specific surface area and interconnected open-framework, which greatly improved the adsorption capacity for organic pollutants. Thanks to the synergism between adsorption-enrichment and in-situ photoelectrocatalytic oxidation, TGH electrodes exhibited efficient photoelectrocatalytic mineralization ability. Moreover, TGH electrodes have excellent stability and the separation-free feature. This work provides new insights into construction of 3D network-structure hydrogels with efficient photoelectrocatalytic mineralization ability and facilitates their potential application for water purification.

\section{Experimental Section}

\subsection{Preparation of samples}

All chemical reagents for synthesis were analytically pure and were used without further purification. The TGH electrode was synthesized by mild chemical reduction at $90^{\circ} \mathrm{C}$ in a quartz mold (as shown in Figure 1a). Specifically, a certain amount of $\mathrm{TiO}_{2}$ nanoparticles (Degussa $\mathrm{P} 25$ grade, $20 \%$ rutile and $80 \%$ anatase) and $75 \mathrm{mg}$ ascorbic acid (Sinoparm, Shanghai) were added into $25 \mathrm{ml} 3.0 \mathrm{mg} / \mathrm{mL}$ homogeneous GO aqueous dispersion under sonication for $30 \mathrm{~min}$. Then, $0.8 \mathrm{ml} 20 \mathrm{mg} / \mathrm{mL}$ dopamine hydrochloride (Sigma-Aldrich) solution was added into the above solution by dripping slowly with continuous stirring. Next, the mixture was transferred into the quartz mold $(5 \mathrm{~cm} * 10 \mathrm{~cm} * 2 \mathrm{~cm})$, where the stainless steel wire mesh $(5 \mathrm{~cm} * 10 \mathrm{~cm})$ was put on the bottom, and sealed well with a quartz plate. Then, the quartz mold was put into water bath and maintained $90^{\circ} \mathrm{C}$ for $30 \mathrm{~min}$. Naturally cooled to room temperature, the black dopamine-functionalized $\mathrm{TiO}_{2}-$ graphenehydrogel electrode was obtained after washing with deionized water (as shown in Figure 1b-c). A 
series of hydrogel electrodes were prepared by changing the weight of $\mathrm{TiO}_{2}(\mathrm{~m}=0 \mathrm{~g}, 0.1125 \mathrm{~g}, 0.1750 \mathrm{~g}$, $0.3000 \mathrm{~g}$ and $0.6750 \mathrm{~g}$ ), which are denoted as GH, TGH-60\%, TGH-70\%, TGH-80\% and TGH-90\%, respectively. The hydrogel electrodes need freeze-drying for further characterization. For comparison, P25 on Ti foil $(5 \mathrm{~cm} * 10 \mathrm{~cm})$ was fabricated using a dip-coating method, the loading amount of P25 was the same as that of TGH-60\%.

\subsection{Characterization}

The morphologies and microstructures of all samples were observed by the field emission scanning electron microscopy (FESEM, Hitachi SU-8010) with an acceleration voltage of $10 \mathrm{kV}$ and the transmission electron microscopy (TEM, Hitachi HT 7700) operated at an accelerating voltage of $100 \mathrm{kV}$. X-ray powder diffraction (XRD) patterns were examined using Rigaku D/max-2400 X-ray diffractometer $(\mathrm{Cu} \mathrm{K} \alpha, \lambda=$ $1.5406 \AA$, 40 kV, $200 \mathrm{~mA}$ ). Raman spectra were carried out using HORIBA HR800 Raman spectrometer with an excitation of $514.5 \mathrm{~nm}$ laser light. BET surface area measurements were recorded by $\mathrm{N}_{2}$ adsorption at $77 \mathrm{~K}$ using a Micrometrics (ASAP 2010V5.02H) surface area analyzer. UV-Vis DRS were got with Hitachi UV-3010 UV-Vis spectrophotometer. The content of $\mathrm{TiO}_{2}$ nanoparticles in $\mathrm{TGH}$ hydrogels were determined by TGA/DSC (Mettler Toledo TGA/DSC 1 ) in air with a heating rate of $10^{\circ} \mathrm{C} / \mathrm{min}$.

\subsection{Photoelectrocatalytic activity}

The photoelectrocatalytic activity of TGH electrodes was measured in a continuous flow reactor(as shown in Figure 4a) and using a conventional three-electrode cell system of CHI 660D electrochemical workstation, in which water was recycled to maintain the reaction temperature at $25^{\circ} \mathrm{C}$, the size of the reactor was $7 \mathrm{~cm} * 12 \mathrm{~cm} * 13 \mathrm{~cm}$. The TGH electrode worked as the anode, platinum plate as the counter electrode, and saturated calomel electrode (SCE) as the reference electrode. A 9 W UV lamp (Cnlight Co., center wavelength $254 \mathrm{~nm}$, light intensity $4.53 \mathrm{~mW} / \mathrm{cm}^{2}$ ) as the UV light source was placed between two working 
electrodes. $10 \mathrm{mg} / \mathrm{L}$ methylene blue (MB) or $5 \mathrm{mg} / \mathrm{L}$ phenol aqueous solution containing $0.1 \mathrm{M} \mathrm{Na}_{2} \mathrm{SO}_{4}$ were used as the simulated wastewater. At given intervals, $7 \mathrm{~mL}$ solution was sampled and could be analyzed without centrifugation. Then, the solution was analyzed by recording the UV-Vis spectrum of MB at the maximum absorbance of $664 \mathrm{~nm}$. HPLC was used to detect the degradation products, and HPLC conditions were shown in supporting information.

\section{Results and Discussion}

\subsection{Fabrication of 3D network structure}

The synthesis route of TGH electrodes was illustrated in Figure 1a (details are shown in the Experimental Section). Figure 1b-c shows the photographs of TGH-80\% electrode. The electrode was a black hydrogel block and exhibited excellent toughness. In order to investigate the effect of dopamine on TGH electrodes, the sample without dopamine was prepared in the same way (labeled rGO). It can be seen from Figure S1 that rGO contracted seriously and was divided into pieces, but TGH electrode exhibited very small volume shrinkage. According to the literatures reported [38, 44-46], that is because of the cross-linking effect generated between dopamine and graphene nanosheets. The 3D network structure of TGH electrodes was further characterized by SEM and TEM. As can be seen from Figure 2a, GH possessed a well-defined and interconnected 3D network structure with pore sizes ranging from 0.1-5 $\mu \mathrm{m}$. Figure S2a presents the TEM image of GH. Some ultrathin graphene nanosheets can be seen clearly, and it has been reported that 3D network-structure GH was formed via the self-assembly of graphene nanosheets with the $\pi-\pi$ stacking and cross-linking effect of dopamine between nanosheets[29, 35]. As shown in the SEM of TGH-80\% (Figure $2 \mathrm{~b}-\mathrm{c})$, the $3 \mathrm{D}$ network structure can still be observed distinctly, and a large number of $\mathrm{TiO}_{2}$ nanoparticles were located on the 3D network-structure matrix of the graphene hydrogel. It could be further seen clearly from Figure S2b and Figure 2d that the $\mathrm{TiO}_{2}$ nanoparticles with a diameter of about 20-50 nm dispersed on 
the surface of graphene nanosheets. Moreover, Figure S3a-c show the SEM images of TGH with different $\mathrm{TiO}_{2}$ contents, with raising $\mathrm{TiO}_{2}$ content, the nanoparticles on the graphene hydrogel were significantly increased, but TGH still remained the 3D network structure.

Then, the structure of TGH was also investigated. Figure 3a shows the $\mathrm{XRD}$ patterns of $\mathrm{TiO}_{2}, \mathrm{GH}$, and TGH. The diffraction peaks of $\mathrm{TiO}_{2}$ fit well with the anatase (JCPDS: 21-1272) and rutile (JCPDS: 21-1276) of $\mathrm{TiO}_{2}$. In the XRD patterns of $\mathrm{TGH}$, no diffraction peak of graphene could be observed, which should be due to a decreased layer-attacking regularity of graphene nanosheets in the TGH. Moreover, the diffraction peaks at about $25.3^{\circ}$ and $27.4^{\circ}$ are gradually moving toward a low angle, revealing the chemical interaction between $\mathrm{TiO}_{2}$ and graphene[15, 47, 48]. Figure $3 \mathrm{~b}$ presents the Raman spectra of $\mathrm{TiO}_{2}, \mathrm{GH}$, and TGH. For $\mathrm{TiO}_{2}$, the peaks at $135 \mathrm{~cm}^{-1}, 187 \mathrm{~cm}^{-1}, 387 \mathrm{~cm}^{-1}, 506 \mathrm{~cm}^{-1}$ and $630 \mathrm{~cm}^{-1}$ correspond to the Raman active modes of anatase $\left(\mathrm{A}_{1 \mathrm{~g}}+2 \mathrm{~B}_{1 \mathrm{~g}}+3 \mathrm{E}_{\mathrm{g}}\right)$ and rutile $\left(\mathrm{A}_{1 \mathrm{~g}}+\mathrm{B}_{1 \mathrm{~g}}\right)$, coinciding with the XRD results above. For $\mathrm{GH}$ and TGH, the peaks at about $1345 \mathrm{~cm}^{-1}$ and $1585 \mathrm{~cm}^{-1}$ correspond to the D- and G-bands of graphene, respectively, indicating the successful reduction of graphene oxide. What's more, with the $\mathrm{TiO}_{2}$ content increasing, the Raman peaks of TGH at about $135 \mathrm{~cm}^{-1}$ shifted to higher wave number, the peaks of D- and G-bands also gradually shifted to lower wave number, which further confirms the chemical interaction between $\mathrm{TiO}_{2}$ and graphene[15, 47].

Next, $\mathrm{N}_{2}$ adsorption/desorption isotherm and pore size analysis were carried out to characterize the specific surface area and pore size distribution of TGH (Figure 3c). The specific surface area of GH was $36.9343 \mathrm{~m}^{3} / \mathrm{g}$, presenting a relatively high value and far higher than that of $\mathrm{rGO}$. And after adding $\mathrm{TiO}_{2}$ nanoparticles, the specific surface area of TGH has been greatly improved (Table S1). TGH- $80 \%$ had the highest value $\left(96.3684 \mathrm{~m}^{3} / \mathrm{g}\right)$, with a 2.5 -fold enhancement compared to that of $\mathrm{GH}$, which extremely benefits to the adsorption-enhancement for organic pollutants, according to the literatures reported[29, 39], the reason 
may be attributed to that $\mathrm{TiO}_{2}$ could partially inhibit the aggregation of graphene nanosheets. The content of $\mathrm{TiO}_{2}$ nanoparticles in TGH hydrogels were determined by TGA/DSC analysis and displaced in Table $\mathrm{S} 1$ and Figure S4, and which is almost close to the theoretical value. From this view, the 3D network-structure TGH electrodes with enhanced specific surface area and interconnected porous channels have the advantage of separation-free feature, high adsorption capacity, which facilitates their potential application for photoelectrocatalytic degradation of organic pollutants in aqueous solution.

\subsection{Synergism between adsorption-enrichment and photoelectrocatalytic oxidation}

Hence, the photoelectrocatalytic degradation activity over TGH electrodes was evaluated using MB and phenol as probe molecules in the continuous flow reactor (Figure 4a), in which liquid flows into the reactor from the bottom right corner and then flows out from the top left corner. Firstly, the dynamic adsorption ability over TGH was studied. As shown in Figure 4b, TGH electrodes exhibited strong adsorptionenrichment for $\mathrm{MB}$, and it took a long time to reach the adsorption saturation over $\mathrm{TGH}$. With the $\mathrm{TiO}_{2}$ content increasing, the adsorption capacity was significantly improved. The high adsorption capacity should be mainly ascribed to the 3D network structure and large specific surface area of TGH. Figure $4 \mathrm{c}$ shows the photoelectrocatalytic degradation of MB over TGH electrodes in the dynamic system. During the degradation process, in the first $5 \mathrm{~h}$, organic pollutant was first adsorbed and enriched onto the TGH, then in-situ degraded drastically, thereafter the degradation reaction reached an equilibrium and the removal rate has always remained constant. Compared to that of P25 on Ti foil, TGH electrodes showed significantly superior photoelectrocatalytic degradation activity, mainly benefiting from their 3D network structure and high specific adsorption-enrichment ability. And among all the TGH electrodes, TGH-80\% exhibited the highest photoelectrocatalytic performance, whose removal rate for MB reached about $65 \%$ when the degradation reaction achieved an equilibrium, the removal rate was more 3 times than that of P25. Next, the effect of bias 
voltage on the photoelectrocatalytic performance was also investigated. As shown in Figure 4d, with improving the bias voltage, the photoelectrocatalytic activity was enhanced gradually. When the bias voltage reached $1.4 \mathrm{~V}$ vs. SCE, TGH- $80 \%$ achieved the highest removal rate about $85 \%$. However, when the bias voltage was further increased, the photoelectrocatalytic performance was reduced gradually, which was mainly ascribed to the fact that the formation of phenol-based polymers and the oxygen evolution reaction occurred on the TGH inhibiting the further degradation of phenol[49, 50]. Interestingly, TGH also exhibited deep mineralization ability for organic pollutants (Figure 4d). It could be seen that the curve trend of TOC (total organic carbon) removal rate was consistent with the degradation plot, and the TOC removal rate for MB over TGH- $80 \%$ at $1.4 \mathrm{~V}$ vs. SCE reached $60 \%$, representing a relatively high value compared to the literature reported[19]. Besides, in the UV-Vis spectra (Figure S5), MB could be drastically degraded and the maximum adsorption peak of MB almost had no shift, further revealing the deep mineralization ability of TGH. At the same time, TGH showed efficient photoelectrocatalytic degradation activity and deep mineralization ability for phenol (Figure 4e), when the degradation reaction achieved an equilibrium, the degradation rate and TOC removal rate reached about $95 \%$ and 50\%, respectively. Moreover, it could be seen from the HPLC spectra (Figure S6) that the peak intensity of both phenol and intermediate species drastically decreased as the reaction proceeded, which indicates that phenol was effectively mineralized. It has been known from the literatures reported that the flow rate of pollutants has a great effect on the degradation activity, because it shortens the contact time between the pollutants and catalysts when the flow rate increases $[19,20]$. As shown in Figure S7, the removal rate of MB was significantly improved with the flow rate decreasing.

According to the above results of photoelectrocatalytic degradation of organic pollutants in a dynamic system, TGH exhibited superior activity than P25, ascribed to the high specific surface area and strong 
adsorption-enrichment ability, which showed a potential application for water purification. As we all know, the stability of catalysts play a key role in the practical application. Therefore, the photoelectrocatalytic stability of TGH was investigated. As can be seen from Figure 5a, TGH-80\% could always keep efficient degradation activity under the continuous running for $100 \mathrm{~h}$, and the structure and morphology remained unchanged, which demonstrates that the TGH possesses excellent photoelectrocatalytic stability. In order to simulate the actual wastewater treatment process, the photoelectrocatalytic degradation was carried out in the multistage series continuous flow reactors (Scheme. S2), in which the wastewater flows continuously through multiple reactors. As shown in Figure 5b, the degradation effect was pretty excellent. When the degradation reaction achieved an equilibrium, the removal rate and mineralization rate of $\mathrm{MB}$ could reach $98 \%$ and $88 \%$, respectively, the color of wastewater flowing out from the reactor was almost identical with that of pure water.

What's more, it could be clearly found from HPLC spectra (Figure S8) that both the MB and intermediate species couldn't be detected nearly when the degradation reaction achieved an equilibrium. The stability test and multistage series degradation data further facilitate the practical application of TGH electrodes for photoelectrocatalytic degradation of wastewater.

\subsection{Photoelectric performance and active species}

It is known that photoelectrocatalytic degradation activity is related to many factors, such as morphology, specific surface area, bang gap, charge-separation property, and active species. Figure S9 presents the UVVis DRS of all samples. The optical absorption of $\mathrm{TiO}_{2}$ was only in the ultraviolet region, and with increasing the graphene content, the absorption of TGH in visible light region was enhanced, but it could be found that the band gap of TGH remained unchanged. According to the previous literature $[26,30,36]$, graphene hydrogel also plays a vital role in inhibiting the recombination of photogenerated electron-hole pairs. As shown in Figure 6a, TGH exhibited dramatically enhanced photocurrent density under UV light irradiation, 
and TGH- $80 \%$ possessed the highest photocurrent density, with a 12 -fold enhancement compared to that of $\mathrm{TiO}_{2}$, which is consistent with the most efficient photoelectrocatalytic activity among all TGH electrodes. The remarkably superior photocurrent density of TGH reveals the extremely fast transfer for photogenerated electrons on graphene hydrogel. However, TGH had no photocurrent response to the visible light. Interestingly, in the comparison test of the photocatalytic, electrocatalytic, and photoelectrocatalytic degradation performance, photoelectrocatalysis showed a significant enhancement of degradation activity, whose removal rate was 2 and 4.7 times higher than that of photocatalysis and electrocatalysis, respectively, which should be attributed to the more efficient charge-separation under the photoelectric synergy. The experiments of active species trapping were carried out using t-butanol (t-BuOH), formic acid $(\mathrm{HCOOH})$ and p-Benzoquinone (p-BQ) as the trapping agent of $\cdot \mathrm{OH}, \mathrm{h}^{+}$and $\cdot \mathrm{O}_{2}^{-}$radials, respectively. It could be seen from Figure $6 \mathrm{c}$ that the photoelectrocatalytic activity both decreased sharply with the addition of t-BuOH and formic acid, but the activity almost had no change with the addition of $\mathrm{p}-\mathrm{BQ}$, which indicates that the $\cdot \mathrm{OH}$ and $\mathrm{h}^{+}$radials worked as the active species.

Based on the above results and discussion, the efficient photoelectrocatalytic mineralization ability of TGH should mainly benefit from the synergism between adsorption-enrichment and in-situ photoelectrocatalytic oxidation and the extremely fast transfer for photogenerated electrons on graphene hydrogel. Accordingly, the photoelectrocatalytic mechanism of TGH electrode was proposed (Figure 6d). Under the synergism between UV light and electric filed, a large number of $\cdot \mathrm{OH}$ and $\mathrm{h}^{+}$radials were generated on the surface of TGH, which effectively oxidized the organic pollutants into $\mathrm{CO}_{2}$ and $\mathrm{H}_{2} \mathrm{O}$.

\section{Conclusion}

In conclusion, $\mathrm{TiO}_{2}$-graphene hydrogel electrodes with $3 \mathrm{D}$ network structure were designed and fabricated successfully via a facile one-pot method. TGH electrodes possess large specific surface area and 
interconnected porous channels, which enables TGH to exhibit strong adsorption-enrichment for pollutants. Interestingly, TGH showed obviously superior photoelectrocatalytic degradation activity for MB and phenol in a dynamic system, the removal rate was 3 times higher than that of P25. In this process, strong oxidizing $\mathrm{h}^{+}$and $\cdot \mathrm{OH}$ radicals worked as the main active groups, which greatly contributed to the deep mineralization ability for organic pollutants over TGH electrodes. The efficient photoelectrocatalytic mineralization ability of TGH electrodes should be mainly ascribed to the synergism between adsorption-enrichment and in-situ photoelectrocatalytic oxidation, and the significant photoelectric performance. Besides, TGH electrodes not only possess the separation-free feature, but also show excellent photoelectrostability. Therefore, 3D network-structure TGH electrodes present vast potential for water purification.

\section{Acknowledgements}

This work was partially supported by the National Basic Research Program of China (973 Program) (2013CB632403), the Chinese National Science Foundation (21437003, 21673126, 21621003), and the Collaborative Innovation Center for Regional Environmental Quality.

\section{References}

[1] L.G. Devi, R. Kavitha, Appl. Catal. B-Environ. 140 (2013) 559-587.

[2] W.-J. Ong, L.-L. Tan, Y.H. Ng, S.-T. Yong, S.-P. Chai, Chem. Rev. (2016).

[3] O. Tomita, T. Otsubo, M. Higashi, B. Ohtani, R. Abe, ACS Catal. 6 (2016) 1134-1144.

[4] D. Liu, J. Wang, X. Bai, R. Zong, Y. Zhu, Adv. Mater. 28 (2016) 7284-7290.

[5] C. Pan, J. Xu, Y. Wang, D. Li, Y. Zhu, Adv. Funct. Mater. 22 (2012) 1518-1524.

[6] C. Yu, G. Li, S. Kumar, K. Yang, R. Jin, Adv. Mater. 26 (2014) 892-898.

[7] D. Xu, B. Cheng, S. Cao, J. Yu, Appl. Catal. B-Environ. 164 (2015) 380-388.

[8] Y. Fan, W. Ma, D. Han, S. Gan, X. Dong, L. Niu, Adv. Mater. 27 (2015) 3767-3773. 
[9] W. Tu, Y. Zhou, Z. Zou, Adv. Funct. Mater. 23 (2013) 4996-5008.

[10] J. Prince, F. Tzompantzi, G. Mendoza-Damián, F. Hernández-Beltrán, J.S. Valente, Appl. Catal. BEnviron. 163 (2015) 352-360.

[11] H. I. Kim, H. N. Kim, S. Weon, G. H. Moon, J. H. Kim, W. Choi, ACS Catal. 6 (2016) 8350-8360.

[12] Y. Ide, N. Inami, H. Hattori, K. Saito, M. Sohmiya, N. Tsunoji, K. Komaguchi, T. Sano, Y. Bando, D. Golberg, Angew. Chem. Int. Edit. 55 (2016) 3600-3605.

[13] M. Cargnello, T.R. Gordon, C.B. Murray, Chem. Rev. 114 (2014) 9319-9345.

[14] W. Jiao, L. Wang, G. Liu, G.Q. Lu, H.-M. Cheng, ACS Catal. 2 (2012) 1854-1859.

[15] Q. Huang, S. Tian, D. Zeng, X. Wang, W. Song, Y. Li, W. Xiao, C. Xie, ACS Catal. 3 (2013) 1477-1485.

[16] A.L. Luna, E. Novoseltceva, E. Louarn, P. Beaunier, E. Kowalska, B. Ohtani, M.A. Valenzuela, H. Remita, C. Colbeau-Justin, Appl. Catal. B-Environ. 191 (2016) 18-28.

[17] M. Zeng, Y. Li, M. Mao, J. Bai, L. Ren, X. Zhao, ACS Catal. 5 (2015) 3278-3286.

[18] B.A. Marinho, R.O. Cristóvão, R. Djellabi, J.M. Loureiro, R.A. Boaventura, V.J. Vilar, Appl. Catal. BEnviron. 203 (2017) 18-30.

[19] W. Jiang, W. Luo, R. Zong, W. Yao, Z. Li, Y. Zhu, Small 12 (2016) 4370-4378.

[20] W. Jiang, Y. Liu, J. Wang, M. Zhang, W. Luo, Y. Zhu, Adv. Mater. Interfaces. 3 (2016) 1500502.

[21] H. Dai, S. Zhang, Z. Hong, X. Li, G. Xu, Y. Lin, G. Chen, Anal. Chem. 86 (2014) 6418-6424.

[22] H.N. Tien, S.H. Hur, J. Colloid Interf. Sci. 437 (2015) 181-186.

[23] A.S. Weingarten, R.V. Kazantsev, L.C. Palmer, M. McClendon, A.R. Koltonow, A.P. Samuel, D.J. Kiebala, M.R. Wasielewski, S.I. Stupp, Nat. Chem. 6 (2014) 964-970.

[24] W. Han, C. Zang, Z. Huang, H. Zhang, L. Ren, X. Qi, J. Zhong, Int. J. Hydrogen Energ. 39 (2014) 19502-19512. 
[25] M. Nawaz, W. Miran, J. Jang, D.S. Lee, Appl. Catal. B-Environ. 203 (2017) 85-95.

[26] Z. Zhang, F. Xiao, Y. Guo, S. Wang, Y. Liu, ACS Appl. Mater. Inter. 5 (2013) 2227-2233.

[27] M. Zhang, W. Jiang, D. Liu, J. Wang, Y. Liu, Y. Zhu, Y. Zhu, Appl. Catal. B-Environ. 183 (2016) 263268.

[28] M. Zhang, W. Luo, Z. Wei, W. Jiang, D. Liu, Y. Zhu, Appl. Catal. B-Environ. 194 (2016) 105-110.

[29] H.-P. Cong, X.-C. Ren, P. Wang, S.-H. Yu, ACS Nano 6 (2012) 2693-2703.

[30] S. Chen, J. Duan, M. Jaroniec, S.Z. Qiao, Angew. Chem. Int. Edit. 52 (2013) 13567-13570.

[31] J. Chen, K. Sheng, P. Luo, C. Li, G. Shi, Adv. Mater. 24 (2012) 4569-4573.

[32] L. Zhang, G. Shi, J. Phys. Chem. C 115 (2011) 17206-17212.

[33] C.J. Madadrang, H.Y. Kim, G. Gao, N. Wang, J. Zhu, H. Feng, M. Gorring, M.L. Kasner, S. Hou, ACS Appl. Mater. Inter. 4 (2012) 1186-1193.

[34] Z. Tang, S. Shen, J. Zhuang, X. Wang, Angew. Chem. 122 (2010) 4707-4711.

[35] Y. Xu, K. Sheng, C. Li, G. Shi, ACS Nano 4 (2010) 4324-4330.

[36] Y. Hou, Z. Wen, S. Cui, X. Feng, J. Chen, Nano Lett. 16 (2016) 2268-2277.

[37] Y. Xiao, J. Zai, X. Li, Y. Gong, B. Li, Q. Han, X. Qian, Nano Energy 6 (2014) 51-58.

[38] H. Gao, Y. Sun, J. Zhou, R. Xu, H. Duan, ACS Appl. Mater. Inter. 5 (2013) 425-432.

[39] Y. Li, W. Cui, L. Liu, R. Zong, W. Yao, Y. Liang, Y. Zhu, Appl. Catal. B-Environ. 199 (2016) 412-423.

[40] M. Zhu, P. Chen, M. Liu, ACS Nano 5 (2011) 4529-4536.

[41] M. Zhu, P. Chen, M. Liu, Langmuir 28 (2012) 3385-3390.

[42] B. Qiu, M. Xing, J. Zhang, J. Am. Chem. Soc. 136 (2014) 5852-5855.

[43] Z. Tong, D. Yang, J. Shi, Y. Nan, Y. Sun, Z. Jiang, ACS Appl. Mater. Inter. 7 (2015) 25693-25701.

[44] L. Li, B. Li, J. Zhang, J. Mater. Chem. A 4 (2016) 512-518. 
[45] X. Song, L. Lin, M. Rong, Y. Wang, Z. Xie, X. Chen, Carbon 80 (2014) 174-182.

[46] C. Cheng, S. Li, J. Zhao, X. Li, Z. Liu, L. Ma, X. Zhang, S. Sun, C. Zhao, Chem. Eng. J. 228 (2013) 468-481.

[47] S.D. Perera, R.G. Mariano, K. Vu, N. Nour, O. Seitz, Y. Chabal, K.J. Balkus Jr, ACS Catal. 2 (2012) 949-956.

[48] X. Chen, F. Liu, B. Liu, L. Tian, W. Hu, Q. Xia, J. Hazard. Mater. 287 (2015) 126-132.

[49] F. Liang, Y. Zhu, Appl. Catal. B-Environ. 180 (2016) 324-329.

[50] Z. Wei, F. Liang, Y. Liu, W. Luo, J. Wang, W. Yao, Y. Zhu, Appl. Catal. B-Environ. 201 (2017) 600-606. 


\section{Figure Captions}

Figure 1. (a) Schematic illustration of synthesis of TGH electrode, (b, c) Photographs of TGH- $80 \%$.

Figure 2. SEM images of (a) GH and (b, c) TGH-80\%. (d) TEM image of TGH-80\%.

Figure 3. (a) XRD patterns, (b) Raman spectra, (c) $\mathrm{N}_{2}$ adsorption/desorption isotherm and pore size distribution plots (inset) of all samples.

Figure 4. (a) Schematic illustration of the continuous flow reactor. (b) The dynamic adsorption curves for MB over different TGH electrodes. (c) Photoelectrocatalytic degradation of MB over different TGH electrodes. (d) Photoelectrocatalytic degradation of MB over TGH-80\% electrode under different bias voltage and TOC removal rate plot at $1.4 \mathrm{~V}$ vs. SCE. (e) Photoelectrocatalytic degradation of phenol over TGH-80\% electrode and TOC removal rate plot.

Figure 5. (a) Photoelectrostability test of TGH-80\% electrode in the continuous flow system and the images of TGH- $80 \%$ before and after degradation (inset). (b) Photoelectrocatalytic degradation of MB over TGH$80 \%$ electrode in the multistage series continuous flow reactors and TOC removal rate.

Figure 6. (a) Photocurrent density of all samples. (b) The comparison test of the electrocatalytic, photocatalytic, photoelectrocatalytic degradation activity. (c) The experiments of active species trapping in degradation of MB over TGH- $80 \%$ electrode in the continuous flow system. (d) Schematic illustration of the possible photoelectrocatalytic mechanism. 

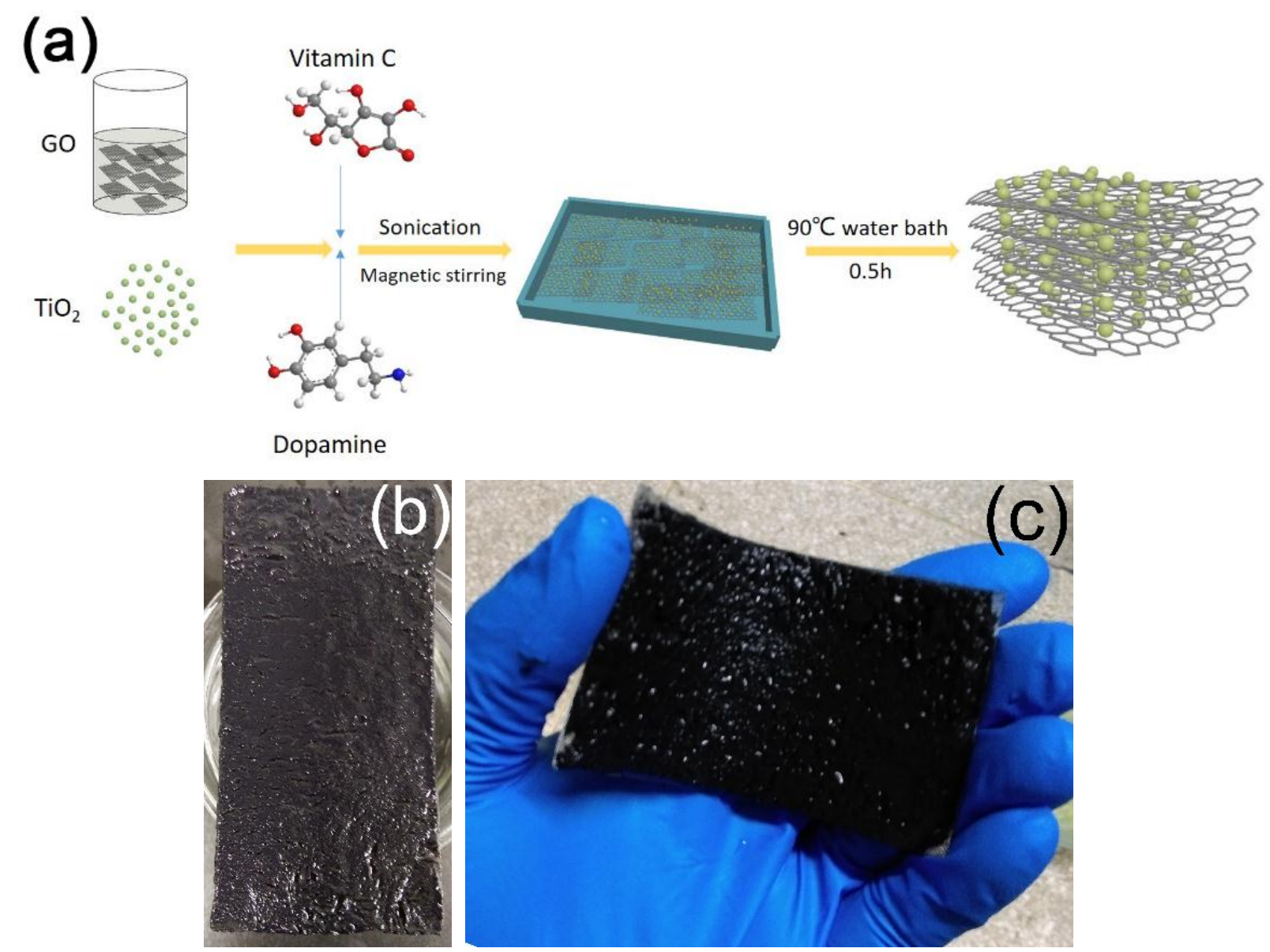

Figure 1. (a) Schematic illustration of synthesis of TGH electrode. (b, c) Photographs of TGH-80\%. 

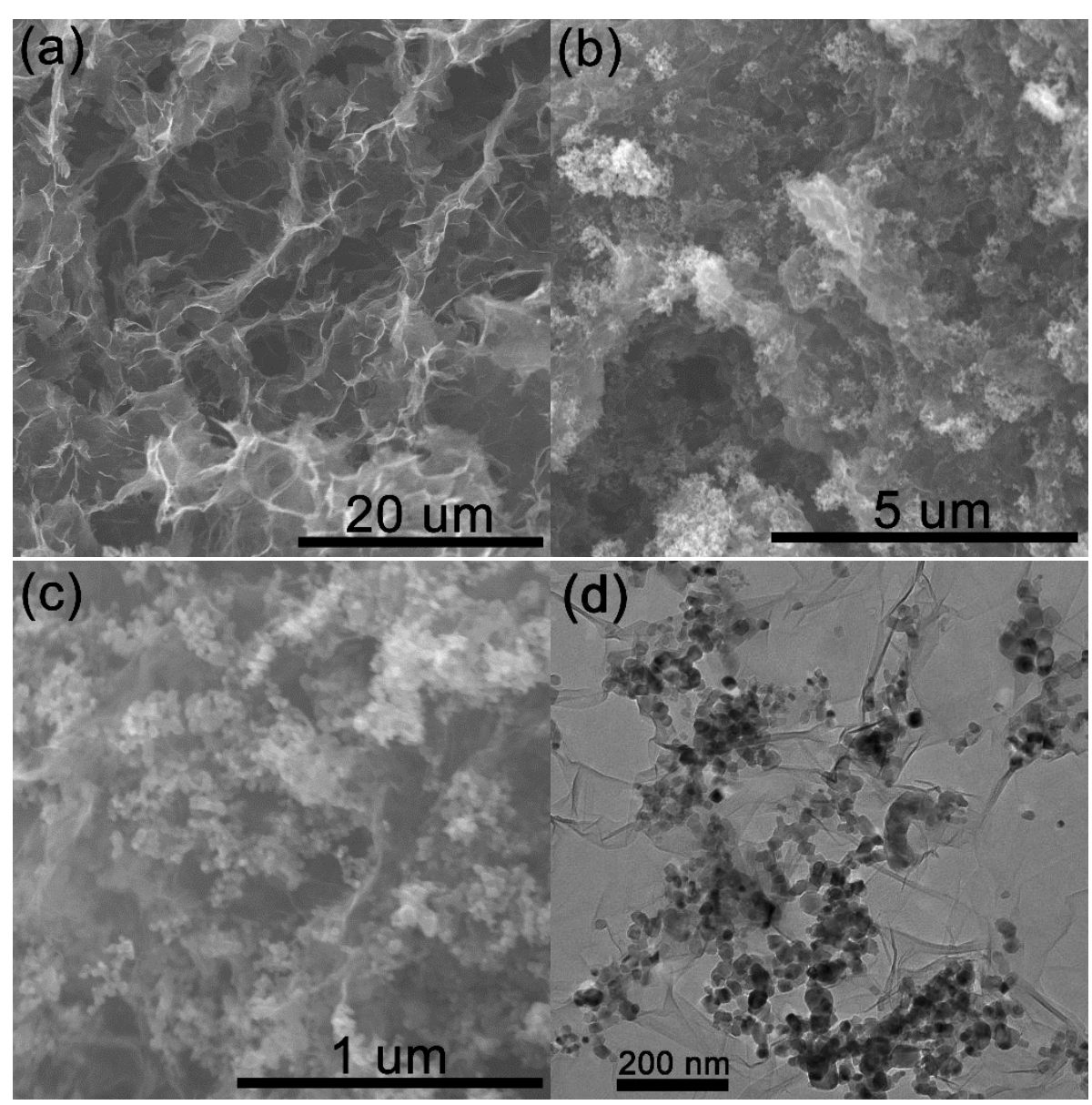

Figure 2. SEM images of (a) GH and (b, c) TGH-80\%. (d) TEM image of TGH-80\%. 

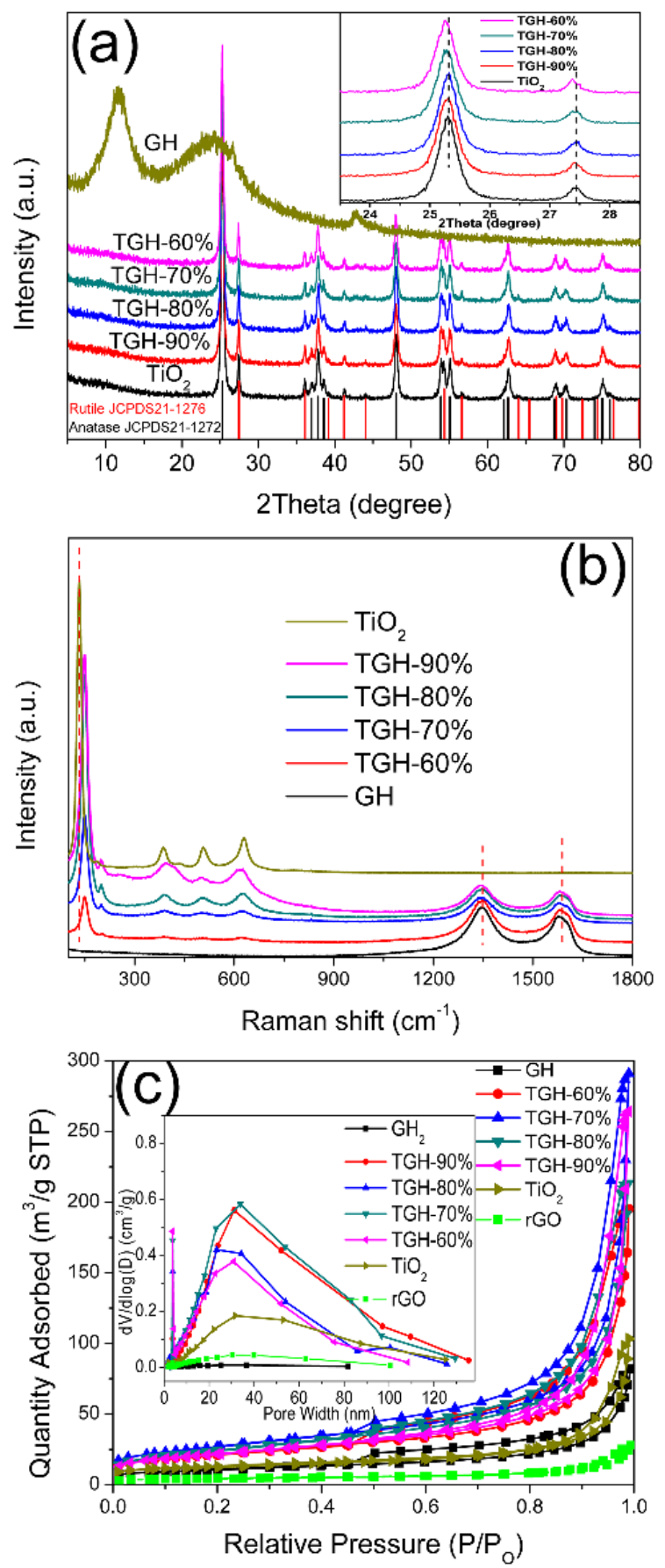

Figure 3. (a) XRD patterns, (b) Raman spectra, (c) $\mathrm{N}_{2}$ adsorption/desorption isotherm and pore size distribution plots (inset) of all samples. 

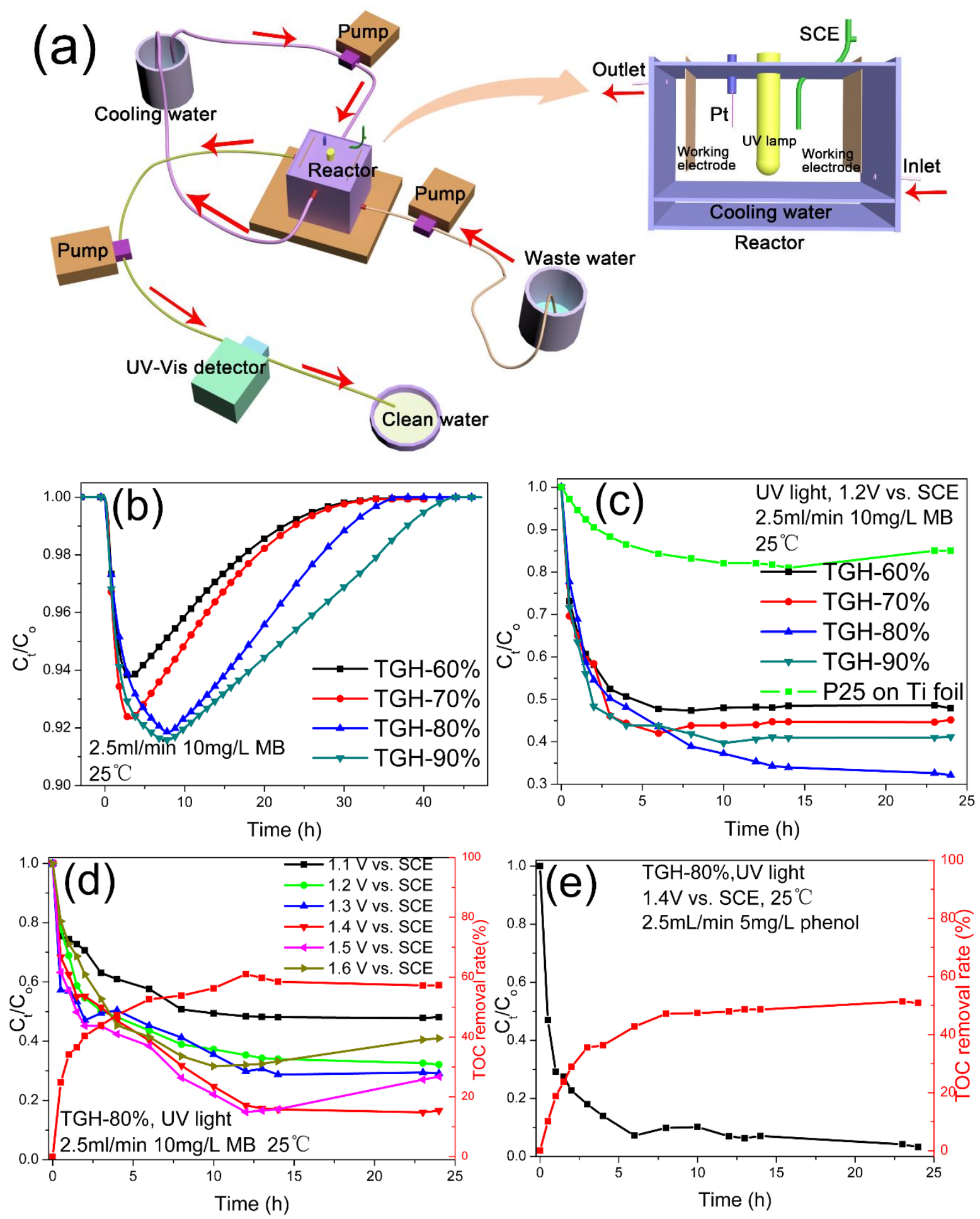

Figure 4. (a) Schematic illustration of the continuous flow reactor. (b) The dynamic adsorption curves for MB over different TGH electrodes. (c) Photoelectrocatalytic degradation of MB over different TGH electrodes. (d) Photoelectrocatalytic degradation of MB over TGH-80\% electrode under different bias voltage and TOC removal rate plot at $1.4 \mathrm{~V}$ vs. SCE. (e) Photoelectrocatalytic degradation of phenol over TGH-80\% electrode and TOC removal rate plot. 

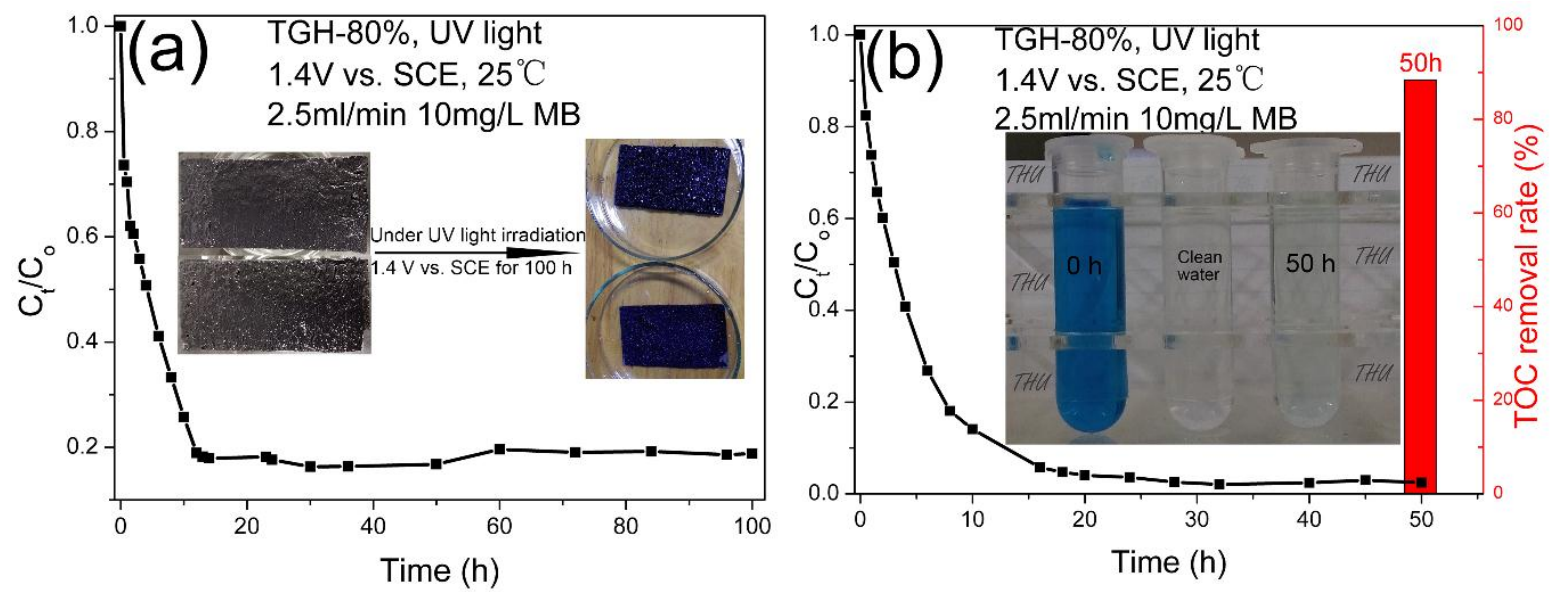

Figure 5. (a) Photoelectrostability test of TGH-80\% electrode in the continuous flow system and the images of TGH-80\% before and after degradation (inset). (b) Photoelectrocatalytic degradation of MB over TGH-80\% electrode in the multistage series continuous flow reactors and TOC removal rate. 

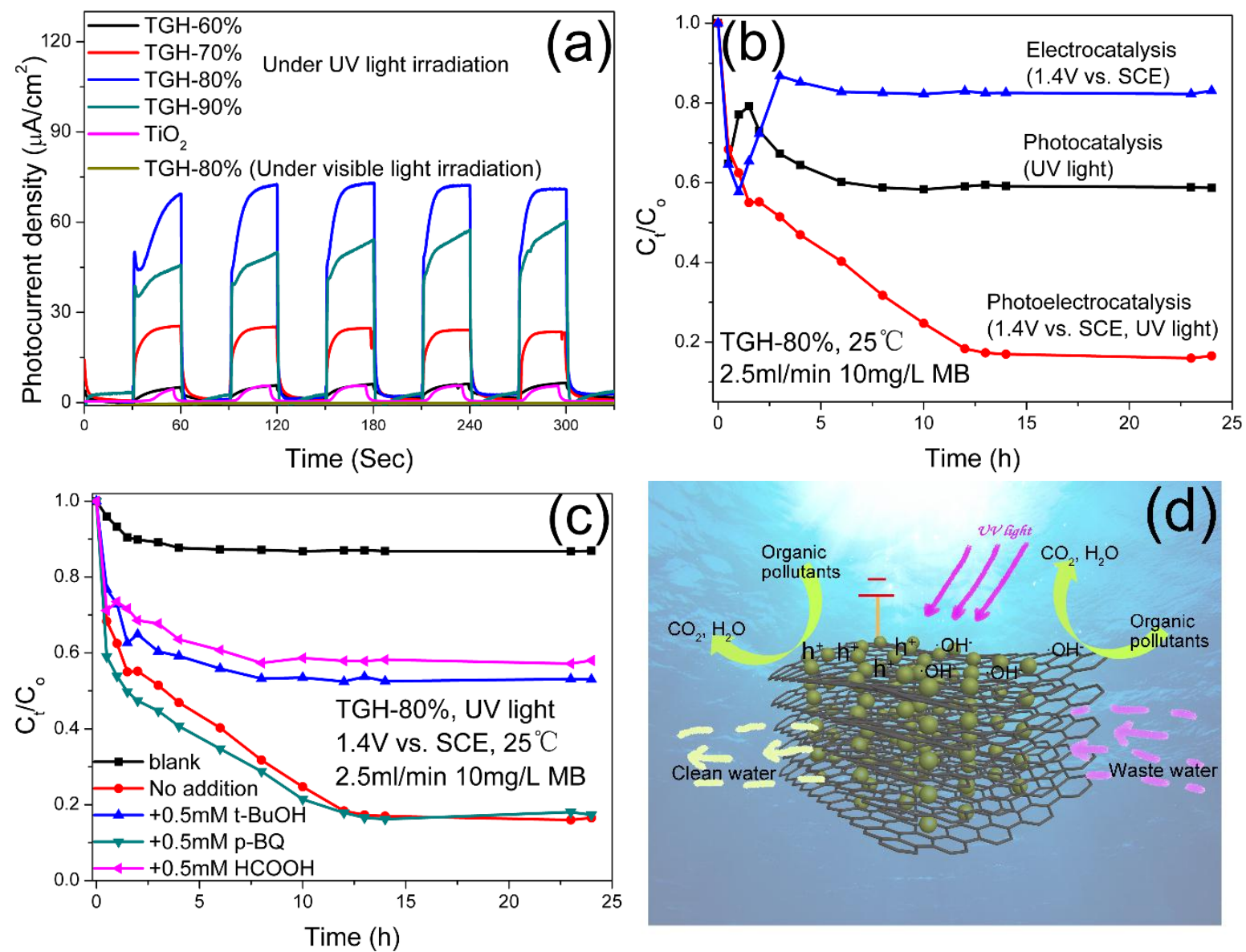

Figure 6. (a) Photocurrent density of all samples. (b) The comparison test of the electrocatalytic, photocatalytic, photoelectrocatalytic degradation activity. (c) The experiments of active species trapping in degradation of MB over TGH-80\% electrode in the continuous flow system. (d) Schematic illustration of the possible photoelectrocatalytic mechanism. 


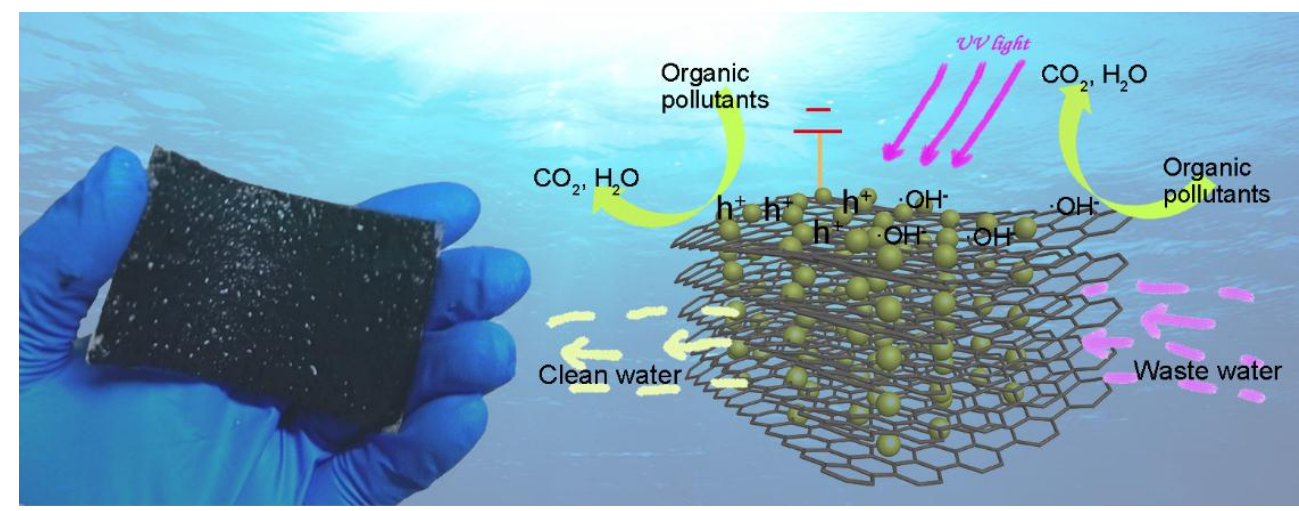

